\title{
Efficient hedging in Bates model using high-order compact finite differences
}

\author{
Bertram Düring and Alexander Pitkin
}

\begin{abstract}
We evaluate the hedging performance of the scheme developed in B. Düring, A. Pitkin, "High-order compact finite difference scheme for option pricing in stochastic volatility jump models", 2017. We compare the scheme's hedging performance to standard finite difference methods in different examples. We observe that the new scheme achieves fourth-order convergence, outperforming a standard, second-order central finite difference approximation in all our experiments.
\end{abstract}

\section{Introduction}

The Bates model [1] can be considered as the market standard in financial option pricing applications. It combines the positive features of stochastic volatility and jump-diffusion models. In this model the option price is given as the solution of a partial integro-differential equation (PIDE), see e.g. [2].

In [4] we have presented a new high-order compact finite difference scheme for option pricing in Bates model. The implicit-explicit scheme is based on the approaches in Düring and Fournié [3] and Salmi et al. [5]. The scheme is fourth order accurate in space and second order accurate in time. It requires only one initial $L U$ factorisation of a sparse matrix to perform the option price valuation. Due to its structural similarities with standard second-order finite difference schemes it can be employed to upgrade existing implementations in a straightforward manner to obtain a highly efficient option pricing code.

Bertram Düring

Department of Mathematics, University of Sussex, Pevensey II, Brighton, BN1 9QH, UK, e-mail: bd80@ sussex.ac.uk

Alexander Pitkin

Department of Mathematics, University of Sussex, Pevensey II, Brighton, BN1 9QH, UK, e-mail: a.h.pitkin@sussex.ac.uk 
In the present work we evaluate the hedging performance of the scheme derived in [4]. We compare the scheme's hedging performance to standard finite difference methods where the new scheme outperforms a standard discretisation, based on a second-order central finite difference approximation, in all our experiments.

This article is organised as follows. In the next section we recall Bates model for option pricing and the related PIDE. We refer here to the [4] paper for the derivation of the implicit-explicit high-order compact finite difference scheme which we adapt and implement to conduct the numerical experiments. Section 3 is devoted to the computation of the so-called Greeks and the evaluation of the scheme's hedging performance in two examples of hedged portfolios.

\section{The Bates Model}

The Bates model [1] is a stochastic volatility model which allows for jumps in returns. Within this model the behaviour of the asset value, $S$, and its variance, $\sigma$, is described by the coupled stochastic differential equations,

$$
\begin{aligned}
& d S(t)=\mu_{B} S(t) d t+\sqrt{\sigma(t)} S(t) d W_{1}(t)+S(t) d J, \\
& d \sigma(t)=\kappa(\theta-\sigma(t))+v \sqrt{\sigma(t)} d W_{2}(t),
\end{aligned}
$$

for $0 \leqslant t \leqslant T$ and with $S(0), \sigma(0)>0$. Here, $\mu_{B}=r-\lambda \xi_{B}$ is the drift rate, where $r \geqslant 0$ is the risk-free interest rate. The jump process $J$ is a compound Poisson process with intensity $\lambda \geqslant 0$ and $J+1$ has a log-normal distribution $p(\tilde{y})$ with the mean in $\log (\tilde{y})$ being $\gamma$ and the variance in $\log (\tilde{y})$ being $v^{2}$, i.e. the probability density function is given by

$$
p(\tilde{y})=\frac{1}{\sqrt{2 \pi} \tilde{y} v} e^{-\frac{(\log \tilde{y}-\gamma)^{2}}{2 v^{2}}} .
$$

The parameter $\xi_{B}$ is defined by $\xi_{B}=e^{\gamma+\frac{v^{2}}{2}}-1$. The variance has mean level $\theta, \kappa$ is the rate of reversion back to mean level of $\sigma$ and $v$ is the volatility of the variance $\sigma$. The two Wiener processes $W_{1}$ and $W_{2}$ have constant correlation $\rho$.

\subsection{Partial Integro-Differential Equation}

By standard derivative pricing arguments for the Bates model, we obtain the PIDE

$$
\begin{array}{r}
\frac{\partial V}{\partial t}+\frac{1}{2} S^{2} \sigma \frac{\partial^{2} V}{\partial S^{2}}+\rho v \sigma S \frac{\partial^{2} V}{\partial S \partial \sigma}+\frac{1}{2} v^{2} \sigma \\
\frac{\partial^{2} V}{\partial \sigma^{2}}+\left(r-\lambda \xi_{B}\right) S \frac{\partial V}{\partial S}+\kappa(\theta-\sigma) \frac{\partial V}{\partial \sigma} \\
-(r+\lambda) V+\lambda \int_{0}^{+\infty} V(S \tilde{y}, v, t) p(\tilde{y}) \mathrm{d} \tilde{y}
\end{array}
$$


which has to be solved for $S, \sigma>0,0 \leq t<T$ and subject to a suitable final condition, e.g. $V(S, \sigma, T)=\max (K-S, 0)$, in the case of a European put option, with $K$ denoting the strike price.

Through the following transformation of variables

$$
x=\log S, \quad \tau=T-t, \quad y=\frac{\sigma}{v} \quad \text { and } \quad u=\exp (r+\lambda) V
$$

we obtain

$$
\begin{aligned}
u_{\tau}=\frac{1}{2} v y\left(\frac{\partial^{2} u}{\partial x^{2}}+\frac{\partial^{2} u}{\partial y^{2}}\right)+\rho v y & \frac{\partial^{2} u}{\partial x \partial y}-\left(\frac{1}{2} v y-r+\lambda \xi_{B}\right) \frac{\partial u}{\partial x} \\
& +\kappa \frac{(\theta-v y)}{v} \frac{\partial u}{\partial y} \lambda \int_{-\infty}^{+\infty} \tilde{u}(x+z, y, \tau) \tilde{p}(z) \mathrm{d} z,
\end{aligned}
$$

which is now posed on $\mathbb{R} \times \mathbb{R}^{+} \times(0, T)$, with $\tilde{u}(z, y, \tau)=u\left(e^{z}, y, \tau\right)$ and $\tilde{p}(z)=$ $e^{z} p\left(e^{z}\right)$. The problem is completed by suitable initial and boundary conditions, which for a European put option are:

$$
\begin{gathered}
u(x, y, 0)=\max (1-\exp (x), 0), \quad x \in \mathbb{R}, y>0, \\
u(x, y, t) \rightarrow 1, \quad x \rightarrow-\infty, y>0, t>0 \\
u(x, y, t) \rightarrow 0, \quad x \rightarrow+\infty, y>0, t>0 \\
u_{y}(x, y, t) \rightarrow 0, \quad x \in \mathbb{R}, y \rightarrow \infty, t>0 \\
u_{y}(x, y, t) \rightarrow 0, \quad x \in \mathbb{R}, y \rightarrow 0, t>0 .
\end{gathered}
$$

\subsection{Implicit-explicit high-order compact scheme}

For the discretisation, we replace $\mathbb{R}$ by $\left[-R_{1}, R_{1}\right]$ and $\mathbb{R}^{+}$by $\left[L_{2}, R_{2}\right]$ with $R_{1}, R_{2}>$ $L_{2}>0$. We consider a uniform grid $Z=\left\{x_{i} \in\left[-R_{1}, R_{1}\right]: x_{i}=i h_{1}, i=-N, \ldots, N\right\} \times$ $\left\{y_{j} \in\left[L_{2}, R_{2}\right]: y_{j}=L_{2}+j h_{2}, j=0, \ldots, M\right\}$ consisting of $(2 N+1) \times(M+1)$ grid points with $R_{1}=N h_{1}, R_{2}=L_{2}+M h_{2}$ and with space step $h:=h_{1}=h_{2}$ and time step $k$. Let $u_{i, j}^{n}$ denote the approximate solution of (2) in $\left(x_{i}, y_{j}\right)$ at the time $t_{n}=n k$ and let $u^{n}=\left(u_{i, j}^{n}\right)$.

For the numerical solution of the PIDE we use the implicit-explicit high-order compact (HOC) scheme presented in [4]. The implicit-explicit discretisation in time is accomplished through an adaptation of the Crank-Nicholson method which includes an explicit treatment for the integral operator. The scheme is fourth-order accurate in space and second-order accurate in time. We refer to [4] for the details of the derivation of the scheme and the implementation of the initial and boundary conditions. 
If not mentioned otherwise, we use the following default parameters in our numerical experiments: $\kappa=2, \theta=0.01, \rho=-0.5, v=0.1, r=0.05, \lambda=0.2$, $\gamma=-0.5$.

\section{The Greeks}

The so-called Greeks are the partial derivatives of the option price with respect to independent variables or parameters. These quantities represent the market sensitivities of options. Practitioners use these quantities to gain an insight into the effects of different market conditions on an options price and furthermore to develop hedging strategies against unfavourable changes in a portfolio of assets.

\subsection{Vega}

Vega measures the sensitivity of the option price with respect to changes in the volatility of the underlying asset, with the volatility given by the square root of the variance, $\sqrt{\sigma}$, i.e.

$$
\text { Vega }=\frac{\partial V}{\partial(\sqrt{\sigma})} .
$$

We examine whether the higher-order convergence achieved in the option price will also be represented in the vega of the option. Vega is calculated from the option price $V(S, \sigma, t)$, while the order of the scheme is maintained by using a fourth-order approximation formula.

$$
\begin{gathered}
\operatorname{Vega}=\frac{\partial V}{\partial(\sqrt{\sigma})}=\frac{\partial y}{\partial(\sqrt{\sigma})} \frac{\partial V}{\partial y} \\
\operatorname{Vega}_{i, j}^{n}=\frac{2 \sqrt{\sigma}}{v}\left(\frac{\partial V}{\partial y}\right)_{i, j}^{n}=\frac{2 \sqrt{\sigma}_{j}}{v} \frac{V_{i, j-2}^{n}-8 V_{i, j-1}^{n}+8 V_{i, j+1}^{n}-V_{i, j+2}^{n}}{12 h}
\end{gathered}
$$

We conduct a numerical study to evaluate the rate of convergence of vega. We refer to both the $l_{2}$-norm error $\varepsilon_{2}$ and the $l_{\infty}$-norm error $\varepsilon_{\infty}$ with respect to a numerical reference solution on a fine grid with $h_{\text {ref }}=0.025$. By fixing the parabolic mesh ratio $k / h^{2}$ we expect these errors to converge as $\varepsilon=C h^{m}$ for some constants $m$ and $C$. We generate a double-logarithmic plot of $\varepsilon$ against $h$ which should be asymptotic to a straight line with slope $m$, with $m$ being the experimentally determined order of the scheme.

As a tool for comparison we perform the same numerical study using a standard second-order central difference scheme. The results of these experiments are seen in Fig. 2 and Fig. 3. We observe here that the experimentally determined convergence 
rates match well the theoretical order of each scheme. The errors at coarse grid, $h=0.4$, are comparable, while on finer grids the high-order compact scheme gives orders of magnitude better accuracy on the same grids, achieving convergence rates of about fourth order.

\subsection{Hedging Vega}

As with all financial trading, options are subject to risk and managing this risk is key to success. One method of managing risk is to establish a hedge against the implied volatility of the underlying asset. This is achieved by creating a vega neutral option position, which will be not be sensitive to fluctuations in volatility.

Fig. 1 Vega of European put option priced under the Bates model with parameters: Strike $K=100$, time to expiry $T=0.5$.

Fig. 2 Convergence of $l_{2}$ error of the vega of a European put option priced under the Bates model with parameters: Strike $K=100$, time to expiry $T=0.5$.
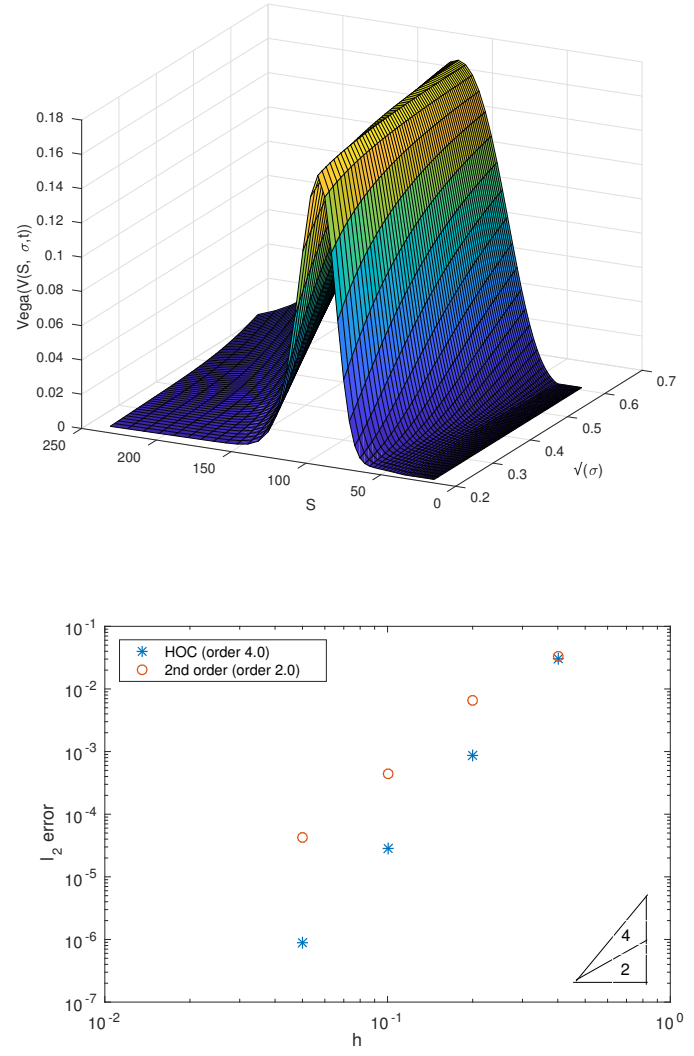


\subsubsection{Hedging Example 1}

An investment fund holds a long position in a non dividend paying stock, XYZ, which is currently trading at $\$ 135$. The investment fund wishes to secure an income from the position and writes some put options for XYZ with strike $\$ 100$. The investment fund now has a position with negative vega. To hedge this vega risk the investment fund creates a ratio vertical put spread by buying put options with strike $\$ 150$, creating a payoff diagram as shown in Fig. 4 .

We propose that using the HOC scheme the investment fund can utilise the highorder convergence in vega to achieve a more accurate vega hedge when constructing the ratio spread. To measure this we compare the ratio used for each mesh size, $h$, with the fine reference grid and examine the resulting percentage error.

The results for the high-order scheme and those for a comparative second-order scheme are shown in Table. 1. The high-order scheme significantly outperforms the second-order scheme at all mesh-sizes, suggesting that when entering a large position the HOC scheme will lead to a significant improvement in the vega hedge.

Table 1 Percentage error in vega hedge ratio

\begin{tabular}{llllll}
\hline Scheme & Mesh-size & Percentage error & Scheme & Mesh-size & Percentage error \\
\hline HOC & 0.4 & 33.3138 & Second-order & 0.4 & 62.0312 \\
HOC & 0.2 & 6.7519 & Second-order & 0.2 & 33.0638 \\
HOC & 0.1 & 0.6251 & Second-order & 0.1 & 7.4073 \\
HOC & 0.05 & 0.0400 & Second-order & 0.05 & 1.5364 \\
\hline
\end{tabular}

Fig. 3 Convergence of $l_{\infty}$ error of the vega of a European put option priced under the Bates model with parameters: Strike $K=100$, time to expiry $T=0.5$.

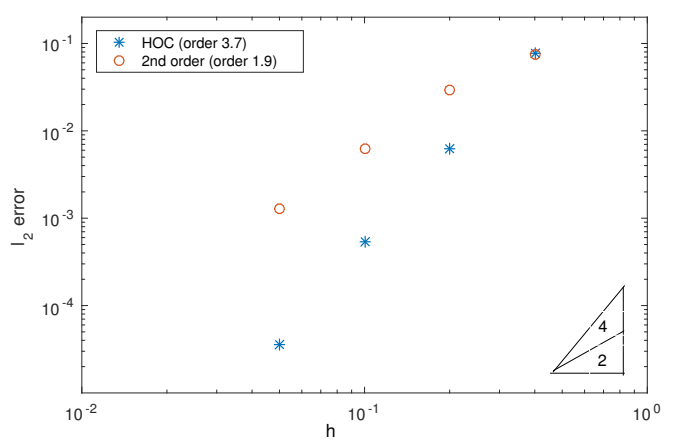




\subsection{Gamma}

Gamma is the second derivative of the option price with respect to the underlying asset. Gamma measures the rate of change in an option's delta, providing information on the convexity of the option's value in relation to the price of the underlying asset,

$$
\Gamma=\frac{\partial^{2} V}{\partial S^{2}} .
$$

We calculate gamma using the option price $V(S, \sigma, t)$. To maintain the order of the scheme we use a fourth-order approximation formula.

$$
\begin{gathered}
\Gamma=\frac{\partial^{2} V}{\partial S^{2}}=\frac{\partial^{2} x}{\partial S^{2}} \frac{\partial^{2} V}{\partial x^{2}} \\
\Gamma_{i, j}^{n}=\frac{1}{S_{i}^{2}}\left(\frac{\partial^{2} V}{\partial x^{2}}\right)_{i, j}^{n}=\frac{1}{S_{i}^{2}} \frac{V_{i-2, j}^{n}-16 V_{i-1, j}^{n}+30 V_{i, j}^{n}-16 V_{i+1, j}^{n}+V_{i+2, j}^{n}}{12 h^{2}}
\end{gathered}
$$

We conduct a numerical study to evaluate the rate of convergence of gamma. We refer to both the $l_{2}$-error $\varepsilon_{2}$ and the $l_{\infty}$-error $\varepsilon_{\infty}$ with respect to a numerical reference solution on a fine grid with $h_{\text {ref }}=0.025$. For comparison we perform the same numerical study using a standard second-order central difference scheme. The results of these experiments are seen in Fig. 6 and Fig. 7.

The HOC scheme achieves convergence rates between three and four for the $l_{2}$ and $l_{\infty}$-errors, respectively. This is an improvement on the second-order scheme and suggests that the high-order scheme is beneficial when developing trading strategies which involve a gamma hedge.

\subsection{Hedging Gamma}

Hedges of gamma risk are often accompanied by a delta hedge, with delta being the first derivative of the option price with respect to the underlying asset. A delta

Fig. 4 Payoff for ratio vertical put spread, examples include a 1:2 spread, where the trader writes two put options then goes long one put option with a higher strike price.

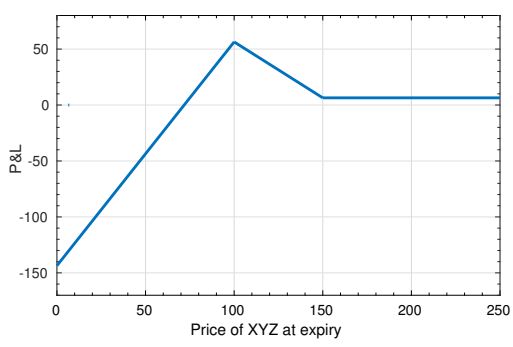


Fig. 5 Gamma of European put option priced under the Bates model with parameters: Strike $K=100$, time to expiry $T=0.5$.

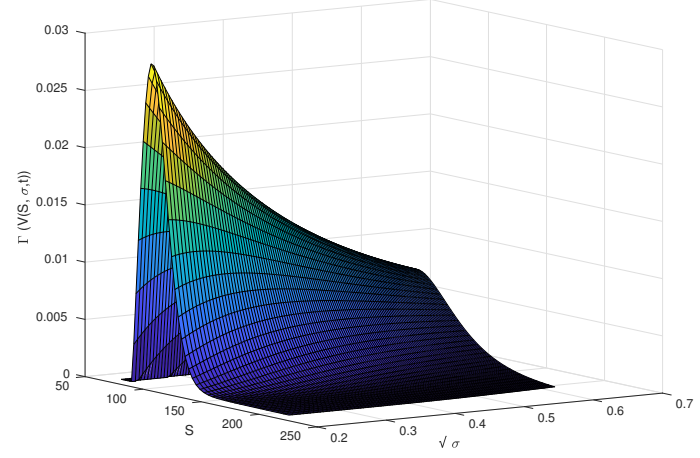

hedged portfolio is not subject to risk owing to a change in the price of the underlying asset, the gamma hedge is a re-adjustment of this delta hedge.

Delta-gamma hedging strategies often require frequent adjustments and hence are subject to high trading costs. However, if executed correctly they can enable the holder to exploit positions with positive theta, meaning the position is profitable over short time durations.

Fig. 6 Convergence of $l_{2}$ error of gamma of a European put option priced under the Bates model with parameters: Strike $K=100$, time to expiry $T=0.5$

Fig. 7 Convergence of $l_{\infty}$ error of gamma of a European put option priced under the Bates model with parameters: Strike $K=100$, time to expiry $T=0.5$.
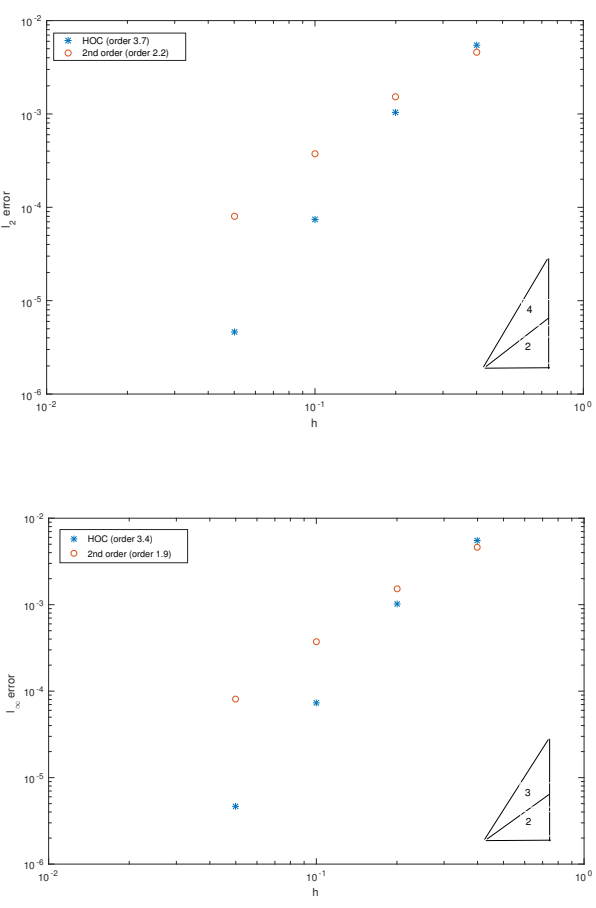


\subsubsection{Hedging Example 2}

An analyst at an investment fund looks to create a strategy with positive theta against the funds currently held assets. They choose a ratio write spread, which involves writing options at a higher strike price than they are purchased. The analyst is wary of the positions risk related to move in the underlying asset and hence adjusts the ratio of short to long options to eliminate the net gamma.

The resulting position will have a delta value which must be hedged before the analyst can assess any profitability from the positive theta of the spread. The delta of the two option positions long and short is totalled and if positive or negative underlying assets are sold or bought, respectively.

The resulting theta is calculated and if positive the analyst can recommend the strategy as a short term trade for the investment fund.

We propose that using the HOC scheme the investment fund can utilise the highorder convergence in gamma to achieve a more accurate gamma hedge ratio. To measure this we compare the ratio used for each mesh size, $h$, with the fine reference grid and examine the resulting percentage error.

The results for the high-order scheme and those for a comparative second-order scheme are shown in Table. 2. The high-order scheme offers better results at all mesh-sizes, this improvement is particularly important in hedged positions which require repeat computation and regular adjustments.

Table 2 Percentage error in gamma hedge ratio

\begin{tabular}{llllll}
\hline Scheme & Mesh-size & Percentage error & Scheme & Mesh-size & Percentage error \\
\hline HOC & 0.4 & 14.8885 & Second-order & 0.4 & 25.1112 \\
HOC & 0.2 & 2.3323 & Second-order & 0.2 & 6.3482 \\
HOC & 0.1 & 0.1281 & Second-order & 0.1 & 1.3304 \\
HOC & 0.05 & 0.0081 & Second-order & 0.05 & 0.2674 \\
\hline
\end{tabular}

Acknowledgements BD acknowledges partial support by the Leverhulme Trust research project grant 'Novel discretisations for higher-order nonlinear PDE' (RPG-2015-69). AP has been supported by a studentship under the EPSRC Doctoral Training Partnership (DTP) scheme (grant number EP/M506667/1).

\section{References}

1. D.S. Bates, Jumps and stochastic volatility: Exchange rate processes implicit Deutsche mark options, Rev. Financ. Stud. 9, 637-654, 1996.

2. R. Cont and P. Tankov, Financial Modelling with Jump Processes, Chapman \& Hall/CRC, Boca Raton, FL, 2004.

3. B. Düring and M. Fournié, High-order compact finite difference scheme for option pricing in stochastic volatility models, J. Comp. Appl. Math. 236, 4462-4473, 2012.

4. B. Düring and A. Pitkin. High-order compact finite difference scheme for option pricing in stochastic volatility jump models. Submitted for publication. arXiv:1704.05308, 2017.

5. S. Salmi, J. Toivanen and L. von Sydow, An IMEX-scheme for pricing options under stochastic volatility models with jumps, SIAM J. Sci. Comp., 36(5), B817-B834, 2014. 